\title{
Crítica de las políticas de evaluación externa de Colombia y Brasil*
}

\author{
Criticism of the \\ External Evaluation \\ Policies of Colombia \\ and Brazil
}

\author{
Crítica das \\ políticas externas \\ de avaliação da \\ Colômbia e do Brasil
}

* Este artículo hace parte de la tesis doctoral Crítica das Políticas de Avaliação Externa na Colômbia e Brasil no contexto da sociedade capitalista del Doctorado en Educación del Programa de Pós-Graduação em Educação (PPGED) de la Universidade Federal de Uberlândia, Minas Gerais, Brasil, como parte del convenio entre la OEA y el Grupo Coimbra de Universidades Brasileras (GCUB), con financiamiento de la Coordenação de Aperfeiçoamento de Pessoal de Nível Superior (CAPES).

** Doctor en Educación de la Universidade Federal de Uberlândia, MG, Brasil. Docente hora cátedra Escuela de Idiomas, grupo de investigación Cultura y Narración en Colombia curnaco, Universidad Industrial de Santander.

Correo institucional: jfremoca@uis.edu.co 


\section{Resumen}

El presente artículo de investigación tiene como objetivo analizar críticamente las políticas de evaluación externa implementadas en Colombia y Brasil, en el contexto de la sociedad capitalista. Con base en este interés, se analizaron documentos oficiales y legales, adoptando la perspectiva de los Estudios Históricos Comparados dentro de la matriz teórico-metodológica del Materialismo Histórico-Dialéctico. Los resultados de investigación demostraron que organismos internacionales al servicio del capital, como el Banco Mundial, ejercen influencia sobre las políticas de evaluación de ambos países mediante las ideologías del mérito y la gestiogracia, que se refleja con la aplicación de exámenes estandarizados, la disposición de indicadores de calidad y la organización de un sistema de responsabilización. A manera de conclusión, se puede afirmar que en ambos sistemas escolares las políticas de evaluación externa vienen comprometiendo los principios de la educación pública mediante la incorporación de la racionalidad mercadológica por intermedio de la competición y la gestión, enmascarando las contradicciones sociales y perpetuando las profundas diferencias individuales y colectivas derivadas de la pobreza y la opresión, resultantes del actual modelo de producción y consumo.

\section{Palabras clave}

política educacional; calidad de la educación; indicadores educativos; responsabilización del docente

Abstract

This article critically analyzes the external evaluation policies implemented in Colombia and Brazil, in the context of capitalist society. Official and legal documents were analyzed, and the chosen perspective was the Comparative Historical Studies within the theoretical-methodological matrix of Historical-Dialectical Materialism. Research results showed that international organizations at the service of capital exert influence on the evaluation policies of both countries through the ideologies of merit and management. As a conclusion it is possible to affirm that in both school systems the policies of external evaluation are compromising the principles of public education by incorporating marketing rationality through competition and management, masking social contradictions and perpetuating the serious individual and group differences derived from poverty and oppression, which are a consequence of the current model of production and consumption.

\section{Keywords}

educational policy; quality of education; educational indicators; teacher responsibility

\section{Resumo}

0 objetivo deste artigo é analisar criticamente as políticas externas de avaliação implementadas na Colômbia e no Brasil, no contexto da sociedade capitalista. Com base nesse interesse, foram analisados documentos oficiais e legais, adotando-se a perspectiva dos Estudos Históricos Comparativos dentro da matriz teóricometodológica do Materialismo Histórico-Dialético. Os resultados da pesquisa mostraram que as organizações internacionais que servem a capital, como o Banco Mundial, exercem influência sobre avaliação de políticas em ambos países pelas ideologias de mérito e gestiogracia, refletidas através da aplicação de testes padronizados, a disposição de indicadores de qualidade e organização de um sistema de responsabilidade. Para concluir, podemos dizer que em ambos sistemas escolares as políticas de avaliação externa estão comprometendo os princípios da educação pública, incorporando a racionalidade mercadológica através da competição e gestão, mascarando contradições sociais e perpetuando profundas diferenças individuais e coletivas derivadas da pobreza e da opressão, resultantes do atual modelo de produção e consumo.

\section{Palavras-chave}

política educacional; qualidade da educação; indicadores educacionais; responsabilidade do professor 


\section{Introducción}

Algunos trabajos académicos demuestran que las evaluaciones estandarizadas no han estado exentas de apreciaciones críticas y de nuevas valoraciones metodológicas. Por un lado, es posible encontrar que este tipo de pruebas develan el carácter autoritario y unilateral de las relaciones humanas al interior de la denominada educación bancaria y, en consecuencia, se afirma que tales exámenes configuran una práctica social violenta y deshumanizante (Remolina, 2013b). En tal sentido, su uso se ha extendido al ámbito de las políticas públicas de control migratorio (Shohamy, 2008), ejerciendo labores discriminatorias (Marbley, Bonner y Berg, 2008) como función de acople entre práctica e ideología (Shohamy, 2007).

Asimismo, evaluaciones internacionales como la prueba Pisa son receptivas al análisis crítico. La relación sujeto-texto, que la Organización para la Cooperación y el Desarrollo Económico (OCDE) propone mediante las denominadas competencias lecto-escritoras, es cuestionada en razón del carácter utilitarista y ahistórico de su base conceptual (Remolina, 2013a). También, es evidente el conflicto generado en culturas consideradas iletradas o analfabetas (Bortoli y Cresswell, 2004), cuyos saberes y modos de aprender difieren de los conocimientos y habilidades requeridos por la sociedad moderna (Dunleavy, 2011).

Por tanto, se destaca la necesidad de reconquistar en general la dimensión humana y democrática de la evaluación como práctica educativa, y particularmente la de las evaluaciones externas (Remolina, 2013b; Tochon, 2011). De igual forma, y no menos importante, se proponen alternativas metodológicas que profundicen su carácter ontológico (Remolina, 2013a). En tal sentido, es posible afirmar que en el marco de una reflexión de la relación sujeto escolar y sociedad, la perspectiva del Materialismo HistóricoDialético (MHD) resulta oportuna para el análisis crítico de las políticas de evaluación. De tal modo, al asumir que las pruebas de evaluación externa van más allá de la simple medición, desmitificar su aparente neutralidad se convierte en asunto de importancia política y educativa.
Para el gobierno colombiano, la evaluación es un mecanismo regulador en la prestación del servicio educativo (Colombia, 2010b). Entre sus funciones está la de mejorar la calidad educativa, además de valorar los avances y garantizar una educación pertinente, significativa y relevante. Razón por la cual se viene implementando un sistema de evaluaciones internas y externas (Colombia, 2010b). No obstante, en el marco socio-político de la implementación del Acuerdo de Paz entre el Gobierno Nacional y el grupo ex-guerrillero de las Farc-EP, las políticas de evaluación externa parecen tomar un matiz especial.

En febrero de 2014, el gobierno colombiano publicó su intención de convertir al país, para el año 2025, en la nación más educada de América Latina. La estrategia: emular los desempeños de los países más destacados en la prueba internacional Pisa (Colombia, 2014a). Así, la calidad educativa como meta y las evaluaciones externas como mecanismo para alcanzarla, asumieron centralidad en la agenda de gobierno. En marzo del año siguiente, el Ministerio de Educación Nacional (Mineducación) hizo público el nuevo sistema de evaluación inspirado en el Sistema Mineiro de Avaliação e Equidade da Educação Pública (Simave) del Estado brasileño de Minas Gerais (Acevedo, 2015). Asimismo, Mineducación presentó el Índice Sintético de Calidad Educativa (Isce), con el cual pretendía medir la calidad de la enseñanza en las Instituciones Educativas (IE) y crear, a su vez, un sistema de incentivos salariales para el profesorado (Sanchez, 2015).

No obstante, la prensa nacional hace eco de las suspicacias originadas en torno a esta nueva forma de medir la calidad educativa en el país. Si bien, la evaluación es fundamental para mejorar la calidad de la enseñanza, no se explica de qué manera las áreas evaluadas por Pisa, referente substancial del Isce, permitan alcanzar los fines Constitucionales de la educación colombiana. Además, si la escuela es factor exclusivo de calidad, según el gobierno, ¿será posible que la suma de atributos cuantitativos, conocidos de antemano por cada IE, sea solución a los problemas educacionales del país? (El Espectador, 2015). 
Ahora bien, siendo Brasil referente de la actual política de evaluación externa en el país, resulta indispensable considerar la influencia de los organismos internacionales sobre la configuración de sus políticas educativas. Si por un lado, (Fonseca, 1998) afirma que la educación brasileña recibió el financiamiento del Banco Mundial (вм) durante los últimos veinte años, por otro, concluye que tal intervención no corrigió de manera significativa los problemas estructurales del sistema escolar básico. Además de eso, no debe olvidarse que tal organismo también impulsó y condujo las reformas educativas de países como México y Chile (Sánchez, 2001).

En resumen, es posible que en Colombia las políticas de evaluación externa hayan asumido centralidad con la incorporación de un concepto heterónomo de calidad y la inclusión de indicadores numéricos. Por tal motivo, y mediante un análisis crítico comparado, resultaría de gran interés identificar qué tipo de ideologías subyacen a la concepción política de las evaluaciones externas tanto de Colombia como de Brasil, dada la influencia de este último sobre el primero, y teniendo en cuenta el predominio de organismos como el BM en la configuración de las políticas educativas latinoamericanas (Bonal, 2002; Sánchez, 2001). Por lo expuesto, el presente trabajo se propone analizar críticamente las políticas de evaluación externa implementadas en los sistemas educativos de ambos países, con lo cual, nuestra pregunta de investigación es: ¿qué tipo de manifestaciones ideológicas estarían presentes en la mediación que ejercen los órganos al servicio del capital sobre la configuración de las políticas de evaluación externa de Colombia y Brasil?

\section{Procedimientos metodológicos}

En el presente trabajo de investigación asumo tres presupuestos epistemológicos, estos son: el Materialismo Histórico-Dialéctico (MHD), la perspectiva de los Estudios Históricos Comparados (EHC) (Franco, 2000; Ciavatta, 2009) y la Totalidad concreta (Kosík, 1995).

De tal manera, adopto las siete fases del MHD (Dussel, 2012): (1) lo real concreto (existente); (2) totalidad caótica; (3) determinaciones abstractas; (4) totalidad construída (concreta); (5) categorías explicativas; (6) totalidad concreta histórica explicada y (7) la realidad conocida.

Igual, se integra la perspectiva de los EHC (Ciavatta, 2009), que permiten la lectura en paralelo sobre realidades distintas, además de mantener coherencia epistemológica con la perspectiva del MHD.

Por lo que se refiere al momento interpretativo, la teoría de las representaciones de Lefebvre (2006) permitió comparar y analizar críticamente la aparente neutralidad de leyes y normas. También se asumió el concepto de ideología como estrategia consciente mediante la cual se disimulan y ocultan las contradicciones de la realidad, presentándola, a su vez, de manera incompleta (Lefebvre, 1975; Lefebvre, 2006; Marx y Engels, 2014).

A partir de estas perspectivas metodológicas, se definieron tres etapas procedimentales: la primera, centrada en la investigación documental y bibliográfica; la segunda, denominada fase descriptiva $y$, por último, la correspondiente al análisis cualitativo de los datos.

En la primera etapa, se seleccionaron documentos relacionados con las políticas públicas de evaluación, correspondientes al sistema educacional básico de cada país. Ahora bien, dado que este tipo de políticas son diacrónicas, se propuso un recorte temporal concentrado sobre dos momentos. En el primero, para el caso de Colombia, se tomó en cuenta los últimos cinco años, es decir, entre 2010 y 2015. Esto en razón a la reciente implementación del modelo evaluativo basado en el indicador Isce. Para el segundo momento, correspondiente a Brasil, se adoptó el período comprendido entre los años 2000 y 2007, ya que en este intervalo aparece el Simave y se da inicio al indicador de calidad brasileño (Índice de Desenvolvimento da Educação Básica - Ideb), que es el referente del nuevo modelo de evaluación colombiano. En definitiva, en esta etapa de investigación documental y bibliográfica, se analizaron los siguientes dispositivos legales por cada país:

En el caso de Colombia, el Decreto 869/2010 que establece el Examen de Estado (Colombia, 2010a); 
la Resolución 15833/2015, que regula las tarifas de matrícula y pensión del servicio educativo de los colegios privados con base en el Isce (Colombia, 2015d); el Boletín Saber, guía metodológica del Isce (Icfes, 2016); la Resolución 503/2014, que define la metodología para la presentación de resultados del Examen de Estado Icfes-Saber $11^{\circ}$ (Icfes, 2014b) y el Decreto 0325/2015, que da inicio al "Día de la Excelencia Educativa" en el sistema escolar colombiano (Colombia, 2015a).

De igual forma, los documentos oficiales correspondientes a Brasil fueron: el Plano Nacional de Educação 2001: Ley 10.172/2001 (Brasil, 2001); la Ley 11.274/2006, que altera los Artículos 29, 30, 32 y 87 de la Ley 9.394, del 20 de diciembre de 1996, que establece directrices y bases de la educación nacional (Brasil, 2006); el Plano de Metas Compromisso Todos pela Educação: Decreto 6.094/2007 (Brasil 2007), que define e implementa el índice Ideb y la Revista da Gestão Escolar (Proalfa/Proeb) (Minas Gerais, 2014), que determina las características más destacadas del Simave.

A su vez, en la segunda etapa procedimental se expusieron las políticas de evaluación externa de ambos países, describiendo cada sistema nacional de evaluación.

Finalmente, en relación con la etapa de análisis, se utilizó como fundamento las fases inductiva y deductiva de la construcción categorial (Paquay, Crahay et Ketele, 2006). Así, se establecieron aproximaciones y distanciamientos comparando críticamente los exámenes estandarizados, los indicadores de calidad, los procesos de participación, la política de responsabilización y la de gestión escolar. Luego, se sintetizaron estas determinaciones abstractas (comunes a ambas realidades) en las siguientes categorías explicativas (MHD: fase 5): calidad educativa, responsabilización docente y participación empresarial. Estas unidades conceptuales se discutieron en la sección Análisis Crítico.

\section{Sistema Nacional de Evaluación}

Los sistemas evaluativos implementados por cada país en general son considerados como elementos propios del nivel macro de la evaluación educacional, el cual tiene que ver con la recolección de información del sistema educativo por parte de organismos exernos a la escuela y cuyo objetivo es verificar la calidad educativa (Afonso, 2009).

En Colombia, el Instituto Colombiano para la Evaluación de la Educación (Icfes) es el organismo responsable de evaluar todos los niveles educativos. Más allá del proceso de preparar y ejecutar exámenes estandarizados a nivel nacional, también se encarga de adelantar investigaciones en torno a la calidad del sistema educativo (Colombia, 2013). En Brasil, el Instituto Nacional de Estudos e Pesquisas Educacionais (Inep) es el responsable directo de organizar e implementar la Prova Brasil, el Sistema de Avaliação da Educação Básica (Saeb) y el Exame Nacional do Ensino Médio (Enem) (Brandalise, 2011).

\section{Evaluación del sistema escolar en Colombia}

Según el Ministerio de Educación colombiano, la evaluación se presenta como mecanismo regulador en la prestación del servicio educativo, ya que las evidencias recolectadas permiten valorar avances y resultados educacionales (Colombia, 2010b). Una mejor educación no está relacionada únicamente con la asistencia o el aumento de la permanencia del alumnado dentro de la escuela (MEN-ICFEs, 2008). El Ministerio relaciona la calidad educativa con la capacidad del sistema de desarrollar en las personas competencias necesarias para un exitoso desempeño ciudadano y productivo.

Así, entre los años 1991 y 1999, el Ministerio de Educación y el Icfes evaluaron de manera muestral y periódica las competencias básicas (Español y Matemáticas) de la población estudiantil de los grados $3^{\circ}, 5^{\circ}, 7^{\circ}$ y $9^{\circ}$ (Colombia, 2007). Sin embargo, las pruebas censales se abrieron paso durante los años 2002 y 2003 cuando fueron aplicados los primeros exámenes para evaluar al alumnado en las áreas de Español, Matemática, Ciencias Naturales y Competencias Ciudadanas (Colombia, 2003; MENICFES, 2008). Según el Ministerio de Educación, resultó claro que la evaluación mejoraría la calidad educativa, por lo cual, fue imprescindible evaluar al 
estudiantado mediante un sistema de pruebas externas más allá de las evaluaciones internas propias del aula de clase (Colombia, 2009a).

\section{Prueba Saber e Indicador Isce}

Inicialmente, el proyecto Saber comprendió la evaluación de quienes finalizaban los grados quinto $\mathrm{y}$ noveno de la Educación Básica Primaria y Secundaria (Colombia, 2007). Gracias a estos resultados, las directivas escolares identificaron habilidades, destrezas y valores en el estudiantado, independiente del lugar de procedencia o las condiciónes sociales, económicas y culturales (Colombia, 2014b). Obtuvieron, asimismo, puntos de referencia respecto a las demás instituciones en el ámbito municipal, departamental y nacional (Colombia, 2003). En el año 2010, con el nombre de Prueba Icfes-Saber $11^{\circ}$, a este proyecto se vinculó el examen para el ingreso a la Educación Superior, antes denominada Prueba de Estado o Prueba Icfes (Colombia, 2010b). Así, a partir de este año, el sistema de evaluaciones externas pasa a llamarse Pruebas Saber.

El Ministerio de Educación estructuró la Prueba Saber en cinco pruebas obligatorias: Lectura Crítica, Matemática, Sociales y Ciudadanas, Ciencias Naturales e Inglés; estas se componen de preguntas abiertas y de opción múltiple (Icfes, 2017). El alumnado es en consecuencia clasificado individualmente mediante la asignación de un puesto, el cual se calcula en función del puntaje obtenido en cada una de los exámenes mencionados. De igual forma, cada IE es clasificada en diferentes categorías de rendimiento (Icfes, 2015).

Por otro lado, respecto al índice Isce, vale decir que este indicador permitió medir cada nivel de enseñanza en una escala numérica de uno a diez (1-10), siendo 10 el máximo valor alcanzado (Colombia, 2015b). Este fue diseñado a finales del 2014 sobre la base del Ideb brasileño y es calculado mediante la suma de cuatro componentes (Icfes, 2016):

a) progreso: refiere cuánto ha mejorado una institución respecto del año anterior;

b) desempeño: expresa el promedio general obtenido en la Prueba Saber correspondiente a las áreas de Matemática y Español evaluadas durante el año en curso;

c) eficiencia: proporción del alumnado aprobado que cada institución reporta al Sistema Integrado de Matrícula (Simat);

d) ambiente escolar: evalúa condiciones adecuadas para el aprendizaje.

El Isce también se utiliza para establecer metas educacionales y como mecanismo de distribución de incentivos salariales. Al estar relacionado con el índice de Mejoramiento Mínimo Anual (MMA), que se calcula con la misma metodología utilizada en Brasil para determinar equivalencias entre exámenes nacionales y la prueba Pisa (Núcleo Educativo San Juan Girón, 2015; Día E Girón Siempre, 2015), el Isce permite identificar aquellas IE con mejores desempeños a fin de destinar el 50\% del salario completo mensual al respectivo personal docente y administrativo (Colombia, 2015c; OECD, 2016).

\section{Evaluación Sistema Educativo Brasil}

Desde hace algunos años las discusiones sobre la evaluación externa en la educación básica brasileña vienen relacionando las escuelas y los sistemas de enseñanza con cuestiones ligadas a la calidad, la medición educacional, la responsabilización y la rendición de cuentas (Neto, 2010). A pesar que los debates y las reflexiones sobre este tipo de evaluaciones hayan sido fuertemente influenciados por la lógica estadounidense (Mezzaroba e Alvarenga, 1999), las iniciativas de exámenes a larga escala en Brasil fueron presentadas como un mecanismo privilegiado de promoción de calidad, acorde con las políticas educacionales globalizadas (Souza e Caetano, 2013). Por tanto, desde hace más de 25 años, las decisiones gubernamentales en torno a la educación recaen sobre tales evaluaciones, cuyo objetivo principal es verificar el desempeño del alumnado mediante pruebas, cuyos resultados son interpretados como evidencia de calidad.

En tal sentido, mediante un sistema técnico nacionalizado que conlleva a la elaboración de cuadros comparativos internacionales y regionales, la evaluación del desempeño estudiantil se consideró 
una expresión educacional fundada en el ideal del desarrollo económico (Sousa, 2013). De acuerdo con esta autora, estos exámenes, además de producirse en un ámbito internacional, son parte de una cultura adoptada por Brasil al ser considerados una práctica educativa propia de los sistemas escolares más avanzados. Es en este contexto que, según la investigadora, los modelos de pruebas internacionales como Pisa se convirtieron en referente de calidad para el sistema educacional brasileño.

En Brasil, la institución responsable de evaluar el sistema educativo es el Inep. Este es un órgano federal vinculado al Ministério da Educação (MEC), encargado de promover estudios e investigaciones sobre el sistema educacional con el fin de subsidiar la formulación e implementación de las políticas educativas (Inep, 2011a).

En 1992, el Inep pasa a tener la responsabilización de coordinar y administrar el desarrollo del Saeb (Bonamino, 2013). Cinco años después, se convirtió en el único organismo responsable por las evaluaciones en todos los niveles de enseñanza, así como de la investigación y la estadística del sistema educacional (Inep, 2011b).

El Saeb y el Enem son dos de las principales evaluaciones realizadas por el Inep. La primera se efectúa cada dos años en la enseñanza fundamental y media. Evalúa de forma muestral el rendimiento estudiantil con base en la educación por competencias, concepto utilizado como referente en los Parámetros Curriculares Nacionales (Barreto, 2013). A su vez se compone de dos procesos evaluativos: la Avaliação Nacional da Educação Básica (Aneb) y la Avaliação Nacional do Rendimento Escolar (Anresc). Esta última, denominada Prova Brasil, de carácter censal, más extensa y detallada, aplicada a estudiantes de $5^{\circ}$ y $9^{\circ}$ año (Inep, 2011e). Con respecto al Enem, es un examen de mayor importancia aplicado por el Inep desde 1997. Este evalúa a la población estudiantil que finaliza el nivel de Enseñanza Medio, sin ser de carácter obligatorio. No obstante, a partir del año 2000 y aunque conserve su naturaleza facultativa, comienza a ser utilizado como prueba de acceso a la universidad (Inep, 2011f).
Por otro lado, en términos de responsabilización y de rendición de cuentas, son identificadas tres generaciones de la evaluación articuladas con la política educacional brasileña (Bonamino, 2013; Brooke, 2013). La primera refiere a las evaluaciones cuyo objetivo es diagnosticar. Su principal tarea consiste en acompañar la evolución de la calidad educativa (Bonamino, 2013). La autora indica que a pesar de la publicación de resultados, estos no son devueltos a cada escuela para su discusión. Al igual que la anterior, las evaluaciones de segunda generación tampoco traían consecuencias materiales para escuelas y docentes. Los resultados obtenidos eran publicados y devueltos a cada institución con efectos meramente simbólicos mediante la divulgación de los llamados rankings escolares. Este tipo de consecuencias simbólicas recibieron el nombre de low-stakes o de consecuencias débiles (Bonamino, 2013; Brooke, 2013).

Por el contrario, las evaluaciones high-stakes, también denominadas de consecuencias fuertes que tipifican una tercera generación, incluían recompensas materiales (Brooke, 2013). Según el autor, estas compensaciones parecían significativas para el individuo y la institución en términos funcionales o económicos, lo que provocaba cambios de comportamiento.

De acuerdo con lo anterior, el Saeb fue considerado una evaluación de primera generación, mientras la Prova Brasil, tanto como la mayoría de exámenes estatales, fue catalogada prueba de segunda generación (consecuencias débiles), esto teniendo en cuenta que más de un tercio de las evaluaciones estatales y longitudinales mostraron disposiciones de tercera generación (fuertes consecuencias) (Bonamino, 2013). En este sentido, es importante señalar que el Saeb no tenía la capacidad de convertirse en referencial de las políticas de responsabilización. Su plano muestral no le permitió identificar las especificidades de municipios y escuelas. En consecuencia, resultaba imposible apoyar con evidencia la formulación de políticas orientadas a la mejora educativa, de forma que la Prova Brasil agregó a la perspectiva diagnóstica del 
Saeb el carácter de los procesos de responsabilización (Bonamino, 2013).

Lo anterior demuestra finalmente que en el sistema educacional brasileño, evaluación y responsabilización van por el mismo camino y tienen objetivos en común.

\section{Simave y el Îndice Ideb}

Minas Gerais históricamente se ha destacado por su experiencia en el campo de la evaluación del aprendizaje. Su pericia viene de tiempo atrás, gracias a la influencia europea durante la década de 1920 y dada la formación de su profesorado en EEUU en los años 50 y 60 . Igualmente, debido al creciente interés que desde inicios de los 90 se originó entorno a la evaluación de la calidad educativa (Vianna, Antunes e Souza, 1993).

Además, la relación de la cultura brasileña con la estadounidense motivó la reproducción de programas de estudio y de prácticas evaluativas (Saul, 2004). Entre las décadas de 1960 y 1970, técnicos estadounidenses, responsables de capacitar al profesorado brasileño, también dirigieron las más importantes recomendaciones educacionales. De igual manera, un significativo número de docentes consiguió efectuar estudios de maestría y doctorado en EEUU. Como consecuencia, las fuentes bibliográficas procedentes de este país influenciaron los cursos universitarios brasileños. Dentro de esta transferencia cultural, los préstamos financieros fueron otro aspecto de consideración e influencia en el ámbito de las políticas públicas educacionales.

Entre 2006 y 2010, Minas Gerais recibió un crédito total de US\$1.607 millones por parte del BM (World Bank Group, 2014). La entidad financiera elogiaba el denominado Choque de Gestão, programa del entonces gobernador Aécio Neves (2003-2010). Esta plataforma político administrativa se basaba en programas prioritarios e indicadores de rendimiento que le permitieron a Minas recuperarse económicamente y mejorar en la prestación de sus servicios (World Bank Group, 2014). Así, en consideración del BM, el estado minero pasó de ser territorio fallido y mal gerenciado, a un Estado fiscalmente responsable.

Tal financiamiento fue ejecutado sobre tres pilares básicos: gestión fiscal, gestión del sector público y desarrollo del sector privado (World Bank Group, 2008). Luego, en una segunda fase denominada Estado para Resultados, se establecieron indicadores de calidad a partir de un sistema de recompensa y castigo (World Bank Group, 2014). Posteriormente, la estrategia gubernamental se focalizó en la participación ciudadana. Fase denominada Estado em Rede, cuyo principal objetivo fue consolidar las fases anteriores y aumentar la eficiencia mediante un modelo ampliamente participativo (World Bank Group, 2014).

En este contexto, surge el Simave con una financiación de US\$150 millones por parte del вM (World Bank Group, 1994). Es así como el sistema de evaluación minero se convirtió en prioridad para la reforma educativa del Estado de Minas que buscaba incorporar reivindicaciones docentes, pero que, al final, terminó integrando políticas administrativas de carácter económico, producto de las orientaciones recibidas por parte de organismos financieros internacionales (Araújo e Silva, 2011).

Por otra parte, cabe resaltar que si bien durante el gobierno de Itamar Franco (1999-2003) el Simave comenzó siendo censal, es en el mandato de Aécio Neves que se convirtió en una prueba de consecuencias fuertes (high-stakes), dada su integración al programa estatal Choque de Gestão, ya que adoptó mecanismos de responsabilización mediante la publicación de resultados escolares y la implementación de premios a la productividad docente a fin de garantizar la calidad educativa en las escuelas mineras (Franco, 2016).

Respecto al índice de calidad brasileño, vale decir que el Ideb se creó en el año 2007 como elemento esencial del programa Plano de Desenvolvimento da Educação (PDE) (Paz e Raphael, 2010). Tal programa del gobierno Lula es lanzado en simultánea con el Plano de Metas Compromisso Todos Pela Educação (РмСТE), que se implementó durante el segundo mandato del Presidente petista (Decreto 
$\left.\mathrm{n}^{\circ} 6.094 / 2007\right)$ y que se convirtió en el proyecto bandera de la política educativa brasileña a través del PDE (Saviani, 2007).

El índice Ideb es un indicador que transita en una escala numérica entre cero y diez $(0-10)$ y es producto de dos factores: la tasa de aprobación y el desempeño promedio en Lengua Portuguesa y Mátemáticas (Saeb para los Estados; Prova Brasil para los municipios) (Inep, 2011c). El Art. 3º del Decreto 6.094/2007 define los alcances y objetivos del Ideb:

A qualidade da educação básica será aferida, objetivamente, com base no Ideb, calculado e divulgado periodicamente pelo Inep, a partir dos dados sobre rendimento escolar, combinados com o desempenho dos alunos, constantes do censo escolar e do Sistema de Avaliação da Educação Básica - Saeb, composto pela Avaliação Nacional da Educação Básica - Aneb e a Avaliação Nacional do Rendimento Escolar (Prova Brasil).

Parágrafo único. O Ideb será o indicador objetivo para a verificação do cumprimento de metas fixadas no termo de adesão ao Compromisso. (Brasil, 2007, p. 5).

De tal modo, el peso del índice intensificó el uso de evaluaciones estandarizadas en todas las redes educativas del país e indujo un patrón de calidad orientado por metas cuantitativas (Barreto, 2013). También, se convirtió en un indicador objetivo para verificar el cumplimiento de las metas del programa Compromisso Todos Pela Educação (CTPE), entre ellas la de alcanzar una media nacional de 6,0 en 2022, año en que se celebra el bicentenario de la Independencia (Inep, 2011d). Esto significa que en el 2021, en términos de desempeño y rendimiento, Brasil debe llegar al promedio actual de los países miembros de la OCDE.

La principal novedad del Ideb consistió en combinar resultados de exámenes estandarizados con datos contextuales, en términos de tasas de abandono y repitencia (Fernandes, 2007). Es decir, este indicador asumió que las escuelas debían ser evaluadas no solo por los procesos de enseñanza y gestión de recursos, sino también por el aprendizaje estudiantil en cuanto capacidades básicas y trayectoria escolar (Soares e
Xavier, 2013). Bajo esta perspectiva, se consideró el aprendizaje (desempeño) y la trayectoria (rendimiento) como elementos substanciales del sistema educacional. De tal manera, fue posible disponer de un índice por cada escuela, Municipio, Estado y el país como un todo (Gatti, 2009).

Es así como el Ideb asumió una importancia capital en Brasil, de forma que su uso se generalizó para diversos fines. Entre ellos: la formulación de políticas públicas educacionales, el planeamiento escolar, la información y el rendición de cuentas (Pontes, 2015). De estos se destaca la implementación de políticas de responsabilización, que dado su carácter neoliberal, la administración Lula trató con prudencia (Vidal e Vieira, 2011), pues el líder sindical, ahora Presidente, había dejado en manos del empresariado el diseño del PDE (Paz e Raphael, 2010; Saviani, 2007), demostrando que el centro supremo de las políticas educativas no era el Estado (Souza, 2014). Tal maniobra le permitió al gobierno aproximarse al bloque económico brasileño, tomando distancia de sus bases partidistas a fin de esquivar enfrentamientos con el poder dominante (Saviani, 2007), para luego terminar gobernando por y para este (Filgueiras, 2006). Así, cobra sentido el hecho que el PDE haya asumido la agenda del movimiento empresarial СTPE (Saviani, 2007).

\section{Análisis crítico}

La calidad de la enseñanza es una de las categorías conceptuales que como síntesis de múltiples determinaciones podría ayudar a explicar la totalidad concreta de las políticas de evaluación externa. En principio, el gobierno de Colombia asegura que la evaluación mejora la calidad educativa. Sobre este argumento plantea la idea de que las Pruebas Saber son instrumento apropiado para alcanzar tal objetivo. Es así como crea el índice Isce que combina resultados de exámenes estandarizados con datos contextuales, tal como el indicador brasileño, a fin de medir el nivel de enseñanza del sistema escolar colombiano. Sin embargo, a diferencia del Ideb, no fue posible encontrar ley, decreto o resolución que definiera e implementara el Isce en Colombia. 
Tampoco pudo identificarse fundamentación teórica alguna. Lo cual da la razón a quienes critican la falta de evidencias conceptuales y empíricas de una función de calidad basada en atributos infundados, añadiendo a esto poca claridad en los procesos de medición y clasificación (Zambrano, 2015).

Por lo anterior, Zambrano concluye que el Isce es una medida foránea, construida de manera unilateral a partir de experiencias internacionales irrelevantes, por tanto, poco confiable; en sus propias palabras “(...) es una medida de productividad académica inscrita dentro de una concepción fabril de la escuela, más no un indicador de calidad educativa." (2015, p. 17).

Cabe entonces preguntar: ¿con qué interés se dispone de un indicador numérico por cada escuela, municipio y región? Tal vez con el propósito de producir cuadros comparativos, buscando emular prácticas internacionales orientadas a la consolidación de estándares de desempeño globalizados. Prueba de ello, el programa Pisa y su influencia en los índices y metas de calidad de ambos países. Sin embargo, debe tenerse en cuenta que tales indicadores, además de promover la definición y verificación de metas cuantitativas, sirven para establecer políticas de responsabilización y de gestión escolar. Categorías conceptuales de importante interés como se verá a continuación.

Para el Banco Mundial, la gestión escolar es parte del proceso de descentralización de los grados de autoridad a nivel escolar, entre los cuales se establece el denominado fundamento de responsabilización (World Bank, 2014). Concepto que en la doctrina neoliberal está vinculado de manera directa al principio de libertad, ya que, refiere a las consecuencias de las acciones del sujeto y se convierte en atributo individual en el afán de pretender mayores rendimientos (Hayek, 2006). Por tanto, en términos educacionales, se considera que la responsabilización es un instrumento de gestión, cuyo propósito es mejorar la educación mediante lógicas de eficiencia y eficacia.

Ahora bien, a pesar de que en Colombia las políticas de responsabilización no están claramente definidas ni materializadas, dada la prematuridad del sistema evaluativo, en Brasil no son asunto menor. La intervención financiera de organismos internacionales en el devenir sociopolítico del Estado de Minas fue determinante para la configuración de las políticas educacionales. A partir de las orientaciones y recomendaciones de carácter económico que el Estado recibió por parte del вм, el Gobierno de Minas implementó dispositivos de responsabilización mediante procesos de gestión, que orientados a la supervisión del funcionario público de manera general, y del profesional docente, particularmente, incluían premios y castigos a la productividad. Así, esta idea fallida que implementada en EEUU perseguía responsabilizar a la escuela pública (Freitas, 2015), produjo efectos laborales e ideológicos en el ámbito escolar brasileño. El rendimiento estudiantil en pruebas externas pasó a ser considerado una responsabilización unilateral docente y se promovieron principios competitivos y meritocráticos (Richter, Souza e Silva, 2015).

Por tanto, las políticas de responsabilización, promovidas por amplios sectores empresariales y apoyadas en evaluaciones de consecuencias fuertes (high-stakes), pretenden de cualquier modo privatizar la educación pública (Emery, 2002). En este sentido, los principios característicos de la ideología de la meritocracia y la competitividad, promocionados mediante este tipo de políticas, hacen parte de la "Privatización encubierta en la educación pública" (Ball y Youdell, 2007). De modo que, competencia y mérito son elementos de un proceso privatizador endógeno, por medio del cual las instituciones educativas tienden a parecer empresas y/o a funcionar como entidades comerciales.

Sin embargo, además de estas ideologías del mérito y la competitividad, se promueve lo que se ha denominado ideología de la gestiocracia, cuyas raíces se hunden hasta la llamada revolución capitalista (Abraham, 2002). Para Abraham, con el avance vertiginoso de la informática, el capitalismo mutó los dispositivos de gestión, haciendo cada vez más necesaria la capacitación de la clase trabajadora, su polifuncionalidad, el control de calidad de los bienes y servicios, el consenso democrático en las 
decisiones, el progreso y la libertad por imposición de la dinámica estructural del propio sistema.

Razón por la cual, la gestión, como conjunto de metodologías, ideologías y prácticas orientadas a la administración y dirección, incorpora también la ideología empresarial en el pensamiento educativo y en las estrategias educacionales de la escuela. Los problemas pedagógicos se convirtieron en problemas administrativos y la eficiencia se transformó en la aplicabilidad de parámetros mensurables, de los cuales hace parte el producto del trabajo evaluado en las pruebas estandarizadas (Marin, Ocampo y Serna, 2005). De ahí que la sobrevivencia escolar comienza a producirse con distintivos democráticos en función de lo que aquí se denomin la ideología gestiocrática.

Cabe recordar que en el presente trabajo, se asume ideología como una elaboración estratégica consciente, cuyo objetivo es disimular y ocultar las propias contradicciones y re-presentar de manera incompleta la realidad, utilizando para ello funciones de disimulación y simulación como si fuese una representación (Lefebvre, 1975; Lefebvre, 2006). En consecuencia, la gestiocracia es ideológica, ya que de forma consciente promueve una reproducción alienada de la gestión bajo los principios de la racionalidad técnico-instrumental que fundamentan la lógica empresarial. De modo que al someter la compleja realidad educativa a la mera cuantificación de sus resultados, las reales contradicciones socioeconómicas son excluidas del ámbito escolar mediante estrategias de disimulación y simulación.

Por otra parte, fue posible observar que mediante la categoría participación el sector empresarial aparece como hilo conductor de la política educacional en ambos países. Desde el PMCTE brasileño se promueve la intervención de amplios sectores económicos en las redes de enseñanza. Se decretan funciones específicas de gestión participativa, tales como delinear y evaluar políticas educacionales, tanto como brindar seguimiento a las metas de evolución del Ideb. Este es el caso del movimiento empresarial Todos pela Educação (TPE). Mientras en Colombia, movimientos como Empresarios por la Educación (EXE, 2010) y
(Todos Por La Educación, 2016) impulsan de igual modo este tipo de iniciativas. Vale destacar que este último, articulado al TPE brasileño, conforma una red internacional de agrupaciones semejantes en otros países de la Región (EXE, 2010).

Sin embargo, llama la atención que en EEUU, país pionero en este tipo de políticas públicas, los resultados obtenidos no superaron las expectativas pregonadas. Bajo el liderazgo de importantes grupos económicos como Business Roudtable, los consejos escolares se redujeron a simples ejecutores gubernamentales; los cabilderos corporativos terminaron por tener influencia directa en la definición de los estatutos educativos y en la contratación de la burocracia educativa, además, con la promoción de las evaluaciones de tercera generación (high-stakes) se eliminó el debate acerca de los fines educativos, se justificó la desagregación del sistema escolar público y se generó un nuevo mercado educacional para beneficio del sector privado (Emery, 2002). En conclusión, denuncia Emery, los objetivos asociados a la democracia y a la lucha contra la segregación se eliminaron y como resultado la labor escolar fue restringida a educar los operarios altamente calificados de la Nueva Economía.

Debe agregarse que tanto en Colombia como en Brasil, la cultura evaluativa se da como estrategia de gobernanza ancorada en lo que aquí se denominó comparativismo globalizador. Se adoptan referentes internos (pruebas nacionales) y externos (prueba Pisa) para ejecutar un tipo de reingeniería educacional cuyo objetivo es aproximar la educación a los intereses del mercado, copiando modelos de otros países y convirtiendo la evaluación en dispositivo de regulación. Es decir, utilizando las críticas de Barriga (2000) al examen escolar, se afirma que las evaluaciones externas aparecen como instrumento globalizante, inversor en las relaciones de poder y saber - tal como denuncia el autor mexicano- con capacidad para transformar problemas sociales en pedagógicos y la potestad de mutar los conflictos epistemológicos educacionales en un asunto técnico evaluativo. 


\section{Consideraciones finales}

En el presente artículo se pretendió analizar críticamente las políticas de evaluación externa implementadas en los sistemas escolares de Colombia y Brasil. Con tal propósito, se adoptó el enfoque de los Estudios Históricos Comparados dentro de la perspectiva epistemológica del Materialismo Histórico Dialéctico.

Las políticas de evaluación externa evidenciaron un fenómeno de doble transferencia en ambos países. En primer lugar, de tipo cultural, propiciado por la influencia de una sociedad sobre otra. Y en segundo lugar, de carácter político, dada la participación de importantes grupos económicos en el delineamiento de la política pública educacional. Sin embargo, este fenómeno es parte de un comparativismo globalizador, práctica internacional cuyo interés es el de estandarizar los fines y desempeños educacionales mediante la implementación de políticas de responsabilización y gestión.

En suma, fueron evidentes dos procesos de regulación. Uno de tipo nacional, efectuado por los grupos económicos de cada país. El otro, de naturaleza transnacional, producto de la intervención de organismos multilaterales como el Banco Mundial y la ocDE a través de sus principales dispositivos de control: préstamos financieros y evaluaciones internacionales (Prueba Pisa). Con lo cual, el Estado deja de ser centro de poder en la definición de las políticas públicas educacionales y conceptos como calidad educativa y evaluación son impuestos bajo la aparente neutralidad de la representación numérica.

En razón a lo anterior y buscando responder la pregunta de investigación, se concluye que las políticas educacionales gestadas y diseminadas por medio de los diversos órganos al servicio del capital, condicionan y determinan en el plano concreto las políticas evaluativas mediante las ideologías de la meritocracia y la gestiocracia. Asimismo, es posible afirmar que en ambos sistemas escolares se construye una lógica empresarial mediante la implementación de políticas de evaluación externa, cuyo interés es el de aproximar la educación a los intereses del mercado y del capital, utilizando mecanismos de calidad, participación y responsabilización.

Así, con las actuales políticas de evaluación externa, la realidad escolar ya no es problematizada, sino juzgada, las contradicciones sociales se enmascaran y el problema de la educación aparece como una interpretación técnica de fácil transferencia, impermeable a las relaciones materiales del entorno, sirviendo, así, a la tarea de ocultar y perpetuar las profundas diferencias individuales y colectivas derivadas de la pobreza y la opresión, resultantes del actual modelo de producción y consumo, cuyo sistema pretende, en todo momento, preservar los privilegios de clase y permitir el acceso a la riqueza a cuenta gotas para quien consigue ascender en la vida dentro de la lógica individualista defendida por neoliberales y neoconservadores.

\section{Referencias}

Abraham, T. (2002). La empresa de vivir. Buenos Aires: Editorial Sudamericana.

Acevedo, T. (2015). Clase aparte. El Espectador. Recuperado de https://www.elespectador.com/opinion/ clase-aparte-columna-551485.

Afonso, A. J. (2009). Avaliação Educacional. Regulação e emancipação: para uma sociologia das políticas avaliativas contemporâneas ( $4^{\text {a }}$. ed.). São Paulo: Cortez.

Araújo, A. B. e Silva, M. A. (2011). O lugar do Sistema Mineiro de Avaliação da Educação Pública (Simave) na busca pela qualidade da educação no Brasil. Roteiro, Joaçaba, SC, 36(2), 205-224.

Ball, S. J. y Youdell, D. (2007). Privatización encubierta en la educación pública. En Internacional de la Educación. V Congreso Mundial, Formas de privatización en la educación y de privatización de la educación (pp. 16-32). Londres: Instituto de Educación, Universidad de Londres.

Barreto, E. S. (2013). Políticas de Currículo e Avaliação e Políticas Docentes. Em Adriana Bauer e Bernardete A. Gatti (orgs.), Vinte e cinco anos de avaliação de sistemas educacionais no Brasil: Implicações nas redes de ensino, no currículo e na formação de professores (pp. 101-117). Florianópolis: Insular.

Barriga, A. D. (2000). Estudio preliminar: El examen: un problema de historia y sociedad. En Ángel Díaz 
Barriga (comp.), El Examen: textos para su historia $y$ debate (pp. 11-28). México D. F.: Plaza y Valdés.

Bonal, X. (2002). Globalización y política educativa: un análisis crítico de la agenda del Banco Mundial para América Latina [Globalization and Education Policy: A Critical Analysis of the World Bank's Agenda for Latin America]. Revista Mexicana de Sociología, (Instituto de Investigaciones Sociales de la Universidad Nacional Autónoma de México), 64(3), 3-35.

Bonamino, A. (2013). Avaliação Educacional no Brasil 25 anos depois: Onde Estamos? Em Adriana Bauer e Bernardete A. Gatti (orgs.), Vinte e cinco anos de avaliação de sistemas educacionais no Brasil: Implicações nas redes de ensino, no currículo e na formação de professores (pp. 43-60). Florianópolis: Insular.

Bortoli, L. y Cresswell, J. (2004). Australias Indigenous Students in PISA 2000: Results from an International Study. Camberwell: Australian Council for Educational Research. (ACER Research Monograph N. ${ }^{\circ}$ 59).

Brandalise, M. Â. T. (2011). Avaliação institucional da escola: conceitos, contextos e práticas. Em xxv Simpósio Brasileiro - II Congresso Iberoamericano da Educação - Jubileu de Ouro da Anpae (19612011), Universidade de São Paulo, Políticas Públicas e Gestão da Educação: construção histórica, debates contemporâneos e novas perspectivas. São Paulo: Associação Nacional de Política e Administração da Educação (ANPAE).

Brasil (2001). Plano Nacional de Educação 2001: Lei 10.172, de 9 de janeiro de 2001. Diário Oficial - 1, Imprensa Nacional, Brasília, DF, Ano CXXXIX (7), 176-197.

Brasil (2006). Lei 11.274, de 6 de fevereiro de 2006. Altera a redação dos arts. 29, 30, 32 e 87 da Lei n. 9.394, de 20 de dezembro de 1996, que estabelece as diretrizes e bases da educação nacional, dispondo sobre a duração de 9 (nove) anos para o ensino fundamental, com matrícula obrigatória a partir dos 6 (seis) anos de idade. Diário Oficial da União, Brasília, D. F., Ano CXLIII (27), 1-2.

Brasil (2007). Decreto n. 6.094, de 24 de abril de 2007. Dispõe sobre a implementação do Plano de Metas Compromisso Todos pela Educação, pela União Federal, em regime de colaboração com Municípios, Distrito Federal e Estados, e a participação das famílias e da comunidade, mediante programas e ações de assistência técnica e financeira, visando a mobilização social pela melhoria da qualidade da educação básica. Diário Oficial da União - Seção 1, Poder Executivo, Brasília, D. F., Ano CXLIV (79), 5-6.

Brooke, N. (2013). Políticas Estaduais de Responsabilização: Buscando o diálogo. Em Adriana Bauer e Bernardete A. Gatti (orgs.), Vinte e cinco anos de avaliação de sistemas educacionais no Brasil: Implicações nas redes de ensino, no currículo e na formação de professores (pp. 119-146). Florianópolis: Insular.

Ciavatta, M. (2009). Estudos comparados: sua epistemologia e sua historicidade, Trab. Educ. Saúde, scielo, Rio de Janeiro, 7 (suplemento), 129-151.

Colombia (2003). Saber para mejorar. Altablero, Bogotá D. C., 24.

Colombia. (2007). Pruebas Saber. Recuperado de http:// colombiaaprende.edu.co/html/home/1592/article-89525.html [18 de noviembre de 2015]

Colombia (2009a). Decreto N. ${ }^{\circ} 1290$ del 16 de Abril de 2009. Por el cual se reglamenta la evaluación del aprendizaje y promoción de los estudiantes de los niveles de educación básica y media. Diario Oficial, Bogotá.

Colombia (2009b). Ley 1324 de 2009. Por la cual se fijan los parámetros y criterios para organizar el sistema de evaluación de resultados de la calidad de la educación, se dictan normas para el fomento de una cultura de la evaluación, en procura de facilitar la inspección y vigilancia del Estado y se transforma el ICFes. Diario Oficial, Bogotá D. C., Año CXLIV (47.409), 11-14.

Colombia (2010a). Decreto N. ${ }^{\circ} 869$ de 2010. Por el cual se reglamenta el Examen de Estado de la Educación Media, ICFES - SABER 11․ Diario Oficial, Bogotá D. C., Año CXLIV (47.655), 4-5.

Colombia (2010b). Evaluación. Bogotá. Recuperado de http://www.mineducacion.gov.co/1621/w3-article-179264.html. [14 de abril de 20]

Colombia (2013). Instituto Colombiano para la Evaluación de la Educación (ICFES). Bogotá. Colombia. Ministerio de Educación Nacional, Bogotá.

Colombia (2014a). Colombia debe trazarse hoy una meta: ser la nación más educada de América Latina en el año 2025. Bogotá. Recuperado de http://tinyurl.com/ y4vqo4uq. [19 de abril de 2015]

Colombia (2014b). Pruebas Saber. Bogotá D. C. Recuperado de http://www.mineducacion.gov.co/1621/w3-article-244735.html [18 de abril de 2015] 
Colombia (2015a). Decreto N. ${ }^{\circ} 0325$ del 25 de febrero de 2015. Por el cual se establece el Día de la Excelencia Educativa en los establecimientos educativos de preescolar, básica y media, y se dictan otras disposiciones. Diario Oficial, Bogotá D. C., (49.436), 221-222.

Colombia (2015b). Índice Sintético. Bogotá D. C. Recuperado de http://aprende.colombiaaprende.edu. co/es/siemprediae/86402 [20 de noviembre de 2015].

Colombia (2015c). MinEducación premiará la excelencia educativa con el "Programa de Incentivos a la Calidad". Centro Virtual de Noticias de la Educación (CVNE) [Comunicado de prensa], Bogotá D. C.

Colombia (2015d). Resolución N. ${ }^{\circ} 15883$ de 2015. Por la cual se establecen los parámetros para la fijación de las tarifas de matrícula y pensiones del servicio de educación preescolar, básica y media prestado por establecimientos educativos de carácter privado para el año escolar que inicia en el 2016. Diario Oficial, Bogotá D. C., (49.649).

Día E Girón Siempre (2015). Mejoramiento Mínimo Anual M.M.A. Recuperado de https://diaegiron.wordpress. com. [11 de abril de 2017]

Dunleavy, J. (2011). Ranking Our Responses to PISA 2009. Education Canada, 51(3).

Dussel, E. (2012). A produção teórica de Marx: um comentário aos Grundrisse (Tradução de José Paulo Netto, $1^{\text {a }}$ ed.). São Paulo: Expressão Popular.

El Espectador (20 de abril de 2015). La educación que medimos. Editorial, Bogotá D. C. Recuperado de http://www.elespectador.com/opinion/editorial/ educacion-medimos-articulo-554187.

Emery, K. (2002). The Business Roundtable and Systemic Reform: How Corporate-Engineered High-Stakes Testing Has Eliminated Community Participation in Developing Educational Goals and Policies (Doctoral dissertation). University of California.

EXE (2010). Informe de gestión 2010. Recuperado de: http:// fundacionexe.org.co/wp-content/uploads/2016/ Biblioteca/INSTITUCIONALES_FExE/infogestion2010.pdf. [25 de noviembre de 2016].

Fernandes, R. (2007). Índice de Desenvolvimento da Educação Básica (Ideb). Brasília, D. F.: Instituto Nacional de Estudos e Pesquisas Educacionais Anísio Teixeira (INEP).

Filgueiras, L. (2006). O neoliberalismo no Brasil: estrutura, dinâmica e ajuste do modelo econômico. Em Eduardo M. Basualdo e Enrique Arceo (orgs.),
Neoliberalismo y sectores dominantes: tendencias globales y experiencias nacionales (pp. 179-210). Buenos Aires: Consejo Latinoamericano de Ciencias Sociales (CLACSO). Recuperado de: http://biblioteca.clacso. edu.ar/clacso/gt/20101101023845/basualdo.pdf

Fonseca, M. (1998). O financiamento do Banco Mundial à educação brasileira: vinte anos de cooperação internacional. Em Livia de Tommasi, Mirian Jorge Warde e Sérgio Haddad (eds.), O Banco Mundial e as políticas educacionais (pp. 229-251). São Paulo: Cortez.

Franco, K. O. (2016). Sistema Mineiro de Avaliação da Educação Pública (sIMAVE): O Estado da Arte da produção científica brasileira (2000-2013) (Dissertação de mestrado). Programa de Pós-Graduação em Educação do Centro de Ciências Humanas e Sociais Aplicadas (CCHSA) da Pontifícia Universidade Católica de Campinas.

Franco, M. C. (2000). Quando nós somos o outro: questões teórico-metodológicas sobre os estudos comparados. Educ. Soc., 21(72), 197-230.

Freitas, L. C. (2015). IDEB: como uma ideia falida influenciou o Brasil. Recuperado de https://avaliacaoeducacional.com/2015/02/02/ideb-como-umaideia-falida-influenciou-o-brasil/. [25 de novembro de 2016]

Gatti, B. (2009). Avaliação de sistemas educacionais no Brasil. Sísifo. Revista de Ciências da Educação, (9), 7-18.

Hayek, F. (2006). Los fundamentos de la libertad (Trad. de José Vicente Torrente). Madrid: Unión Editorial. Icfes

Icfes (2014). Resolución No 503 de 2014. Por la cual se establece la metodología para la presentación de resultados en el examen de Estado para la Educación Media Icfes Saber $11^{\circ}$, para la selección de estudiantes con mejores resultados, para la Clasificación de los Establecimientos Educativos y sus sedes y se adoptan otras determinaciones. Diario Oficial, Año CL (49.221), 69-71.

Icfes (2015). Clasificación de planteles. Bogotá D.C. Recuperado de http://www2.icfes.gov.co/instituciones-educativas-y-secretarias/saber-11/clasificacionde-planteles . [19 de noviembre de 2015]

Icfes (2016). ISCE: Guía metodológica. Boletín Saber en Breve, Bogotá D.C., n. ${ }^{\circ} 5$.

Icfes (2017). Información general del examen Saber $11^{\circ}$. Bogotá D.C. Recuperado de http://www2.icfes.gov. co/estudiantes-y-padres/saber-11-estudiantes/informacion-general-del-examen [15 de marzo de 2019]. 
Inep (2011a). Conheça o Inep. Brasília, D. F. Recuperado de http://portal.inep.gov.br/conheca-o-inep. [2 de setembro de 2015]

Inep (2011b). História. Brasília, D. F. Recuperado de http:// portal.inep.gov.br/historia. [11 de abril de 2015].

Inep (2011c). Metodologia da concepção do Ideb (Nota técnica). Brasília, D. F. Recuperado de http://portal. inep.gov.br/web/guest/ideb [31 de agosto de 2015]

Inep (2011d). O Pisa e o Ideb. Brasília, D. F. Recuperado de http://portal.inep.gov.br/internacional-novo-pisaopisaeideb. [14 de abril de 2015]

Inep (2011e). Sobre a Anresc (Prova Brasil). Brasília, D. F. Recuperado de http://portal.inep.gov.br/educacaobasica/saeb/sobre-a-anresc. [13 de setembro de 2016].

Inep (2011f). Sobre o Enem. Brasília, D. F. Recuperado de http://tinyurl.com/y3e5jg6z. [1 de dezembro de 2015]

Kosík, K. (1995). Dialética do concreto (Trad. de Célia Neves e Alderico Toríbio, revisão de Célia Neves, $2^{\text {a }}$. ed.). Rio de Janeiro: Paz e Terra.

Lefebvre, H. (1975). Lógica formal Lógica dialética (Trad. de Carlos Nelson Coutinho). Rio de Janeiro: Civilização Brasileira.

Lefebvre, H. (2006). La Presencia y la Ausencia: Contribución a la teoría de las representaciones (Trad. de Óscar Barahona e Uxoa Doyhamboure). México D. F.: Fondo de Cultura Económica.

Marbley, A. F., Bonner, F. y Berg, R. (2008). Measurement and Assessment: Conversations with Professional People of Color in the Field of Education. Multicultural Education, 16(1), 12-20.

Marin, L. F., Ocampo, J. J. y Serna, E. (2005). Crítica y Educación: Ensayos sobre las reformas educativas en Colombia. Armenia: Fussion Creativa.

Marx, K. y Engels, F. (2014). La ideología alemana: Crítica de la novísima filosofía alemana en las personas de sus representantes Feuerbach, B. Bauer y Stirner, y del socialismo alemán en las de sus diferentes profestas. Madrid, España: Ediciones AKAL.

MEN-ICFES (2008). Evaluaciones Externas en América Latina: Caracterización de los procesos logísticos utilizados para la aplicación de pruebas censales y muestrales en Cuba, Chile, Argentina, México, Uruguay y Colombia. El caso Colombia (1 ${ }^{\text {a }}$. ed.). Bogotá D. C.: Logoformas S. A. (Estudio sobre evaluación educativa), Universidad de San Buenaventura, Cali, Grupo de Investigación en Evaluación y Calidad de la Educación (GIECE), Semillero de Investigación en Evaluación y Calidad de la Educación (SIECE) y Centro de Investigaciones Boaventuriano (CIB).

Mezzaroba, L. e Alvarenga, G. M. (1999). A trajetória da avaliação educacional no Brasil. Em Georfravia Montoza Alvarenga (orgs.), Avaliar um compromisso com o ensino aprendizagem (pp. 29-81). Londrina: Núcleo de Estudos e Pesquisa em Avaliação Educacional.

Minas Gerais (2014). simave. Sistema Mineiro de Avaliação da Educação Pública: Proalfa/Proeb. Revista da Gestão Escolar, Belo Horizonte. Recuperado de: http://www.simave.caedufjf.net/ wp-content/uploads/2015/06/SIMAVE-RGE-WEB. pdf . [21 de septiembre de 2016].

Neto, J. L. H. (2010). Avaliação externa de escolas e sistemas: questões presentes no debate sobre o tema. Revista Brasileira de Estudos Pedagógicos, 91(227), 84-104.

Núcleo Educativo San Juan Girón (2015). Día E de la Excelencia Educativa. Recuperado de https://competenciasciudadanasgiron.wordpress.com/dia-e-de-laexcelencia-educativa/. [25 de septiembre de 2016].

OECD (2016). La educación en Colombia: Revisión de políticas nacionales de educación. París: OECD.

Paquay, L., Crahay, M. et Ketele, J.M. de (2006). $L$ 'analyse qualitative en éducation: des pratiques de recherche aux critères de qualité. Bruxelles: De Boeck Université.

Paz, F. M. e Raphael, H. S. (2010). O IDEB e a qualidade da educação no ensino fundamental: fundamentos, problemas e primeiras análises comparativas. Revista omNia Humanas, 3(1), 8-30.

Pontes, L. A. F. (2015). Medidas de eficácia escolar no contexto das políticas brasileiras de responsabilização educacional: o caso do Índice de Desenvolvimento da Educação Básica, o IDEB, em Minas Gerais (Dissertação de Doutorado em Educação). Programa de Pós-graduação em Educação, na área de Gestão, Políticas Públicas e Avaliação Educacional, da Universidade Federal de Juiz de Fora.

Remolina, J. F. (2013a). La lectura en Paulo Freire y la competencia lectora de PISA. Educação, 36(2), 223-231. 
Remolina, J. F. (2013b). Las evaluaciones estandarizadas desde una vision freireana. Revista Eletrônica de Educação, 7(1), 369-382.

Richter, L. M., Souza, V. A. e Silva, M. V. (2015). A dimensão meritocrática dos testes estandardizados e a responsabilização unilateral nos docentes. Revista Brasileira de Política e Administração da Educação, 31(3), 607-625.

Sanchez, C. M. (2015). Nuestros hijos pueden ser los más educados de Latinoamerica: Los profesores serán premiados con un salario adicional al año si cumplen sus metas propuestas hoy. El Tiempo, Bogotá. Recuperado de https:/www.eltiempo.com/archivo/ documento/CMS-15460955. [25 de marzo de 2015].

Sánchez, M. (2001). Influencia del Banco Mundial y la CEPAL en las tendencias educativas recientes en algunos países latinoamericanos. Revista Latinoamericana de Estudios Educativos, XXXI(4), 55-97.

Saul, A. M. (2004). Evaluación del aprendizaje: una vía para el mejoramiento de la calidad en la escuela. En Isabel Franchi Cappelletti (coord.), Evaluación Educativa: Fundamentos y prácticas ( $1^{\text {a }} \mathrm{ed}$. en español). Mexico D. F.: Siglo XXI Editores.

Saviani, D. (2007). O Plano de Desenvolvimento da Educação: análise do projeto do MEc. Educ. Soc., 28(100), 1231-1255.

Shohamy, E. (2007). Language Tests as Language Policy Tools. Assessment in Education, 14(1), 117-130.

Shohamy, E. (2008). Language Policy and Language Assessment: The Relationship. Current Issues in Language Planning, 9(3), 363-373.

Soares, J. F. e Xavier, F. P. (2013). Pressupostos educacionais e estatísticos do IDEB. Educação \& Sociedade, 34(124), 903-923.

Sousa, S. Z. (2013). Avaliação Externa e em Larga Escala no Âmbito do Estado Brasileiro: Interface de experiências estaduais e municipais de avaliação da educação básica com iniciativas do governo federal. Em Adriana Bauer e Bernardete A. Gatti (orgs.), Vinte e cinco anos de avaliação de sistemas educacionais no Brasil: Implicações nas redes de ensino, no currículo e na formação de professores (pp. 61-85). Florianópolis: Insular.

Souza, S. A. e Caetano, M. R. (2013). Redes de Relações e o Instituto Ayrton Senna. Em Theresa Adrião e Vera Peroni (orgs.), Gestão Municipal da Educação e as Parcerias com o Instituto Ayrton Senna (pp. 98-125). Recife: Anpae.
Souza, V. A. (2014). O Plano de Metas Compromisso Todos Pela Educação: desdobramentos na gestão educacional local e no trabalho docente (Dissertação de Doutorado em Educação). Programa de Pós-graduação em Educação da Faculdade de Educação da Universidade Federal de Uberlândia.

Tochon, F. V. (2011). Le savoir-évaluer comme politique éducative: vers une évaluation plus profonde. Mesure et évaluation en éducation, 34(3), 133-156.

Todos Por La Educación (2016). Recuperado de http:// todosporlaeducacion.co/co/somos/. [5 de abril de 2017]

Vianna, H. M., Antunes, A. L. e Souza, M. A. (1993). Desenvolvimento de um programa de avaliação do sistema estadual de ensino: o exemplo de Minas Gerais. EAEE Estudos em Avaliação Educacional, (8), 5-37.

Vidal, E. M. e Vieira, S. L. (2011). Gestão educacional e resultados no IDEB: um estudo de caso em dez municípios cearenses. EAEE Estudos em Avaliação Educacional, 22(50), 419-434.

World Bank (2014). Economics of Education. Brief Education, Washington, DC: The World Bank Group.

World Bank Group (1994). Brazil - State of Minas Gerais: Basic Education Quality Improvement (ProQualidade). Washington, DC: The World Bank Group.

World Bank Group (2008). Brazil - Minas Gerais Partnership for Development Project: Report N. ${ }^{\circ}$ ICR0000845. Washington, DC: The World Bank Group.

World Bank Group (2014). Brazil - Third Minas Gerais Development Partnership Development Policy Loan Project. Report N. ${ }^{\circ}$ ICR00002866. Washington, DC.: The World Bank Group.

Zambrano, M. F. (2015). Alcances e inconsistencias del Indice Sintético de Calidad Educativa (ISCE), diseñado y aplicado por el Ministerio de Educación de Colombia en el año 2015. Bogotá: Fundación IQ-Matrix Iniciativas para la sociedad del conocimiento. 